\title{
Histoplasmosis diseminada con lesiones cutáneas y osteomielitis en un niño con Leucemia Linfoblastica Aguda en mantenimiento
}

\section{Disseminated histoplasmosis with skin lesions and osteomyelitis in a child with acute Lymphoblastic Leukemia Undergoing maintenance treatment}

\author{
Irene Benítez', Mónica Rodríguez', Verónica Lezcano', Zoilo Morel², José Pereira³, Silvia
} Brizuela $^{4}$, Humberto Galleano ${ }^{5}$

\begin{abstract}
RESUMEN
La histoplasmosis es una micosis causada por el hongo Histoplasma capsulatum y generalmente se manifiesta como una infección diseminada en pacientes inmunocomprometidos. La osteomielitis por H. capsulatum es rara y difícil de diagnosticar. Presentamos un caso inusual de histoplasmosis diseminada, causada por H. capsulatum, con lesiones en piel, mucosas y osteomielitis en un niño de 10 años de edad con Leucemia Linfoblastica Aguda en tratamiento con metotrexato vía oral a dosis baja, con adecuada respuesta a Anfotericina b y mantenimiento con Itraconazol.
\end{abstract}

Palabras claves: Histoplasmosis, osteomielitis, inmunocomprometidos, metotrexato, niños.

\begin{abstract}
Histoplasmosis is a mycosis caused by the fungus Histoplasma capsulatum and usually manifests as a disseminated infection in immunocompromised patients. Osteomyelitis due to $H$. capsulatum is rare and difficult to diagnose. We present an unusual case of disseminated histoplasmosis, caused by $H$. capsulatum, with lesions in skin, mucous membranes and osteomyelitis in a 10-yearold boy with acute Lymphoblastic Leukemia Undergoing low-dose oral methotrexate treatment, who had an adequate response to Amphotericin B and maintenance treatment with Itraconazole.
\end{abstract}

Keywords: Histoplasmosis, os teomyelitis, immunocompromised, methotrexate, children.

\section{INTRODUCCIÓN}

La histoplasmosis se define como la infección causada por el hongo dimorfo Histoplasma capsulatum. El mismo se encuentra principalmente en suelos que contienen grandes cantidades de excrementos de aves o guano de murciélago ${ }^{(1,2)}$.
La infección con H. capsulatum se desarrolla cuando microconidios o pequeños elementos de hifas se inhalan y se convierten en levaduras en los pulmones, o cuando los organismos en focos de infección quiescentes previos son reactivados durante una inmunosupresión ${ }^{(3)}$.

\footnotetext{
${ }^{1}$ Servicio de Infectología Pediátrica, Hospital Central del Instituto de Previsión Social. Asunción. Paraguay ${ }^{2}$ Servicio de Reumatología, Hospital Central del Instituto de Previsión Social. Asunción. Paraguay.

${ }^{3}$ Programa Nacional de Control de la Lepra, Ministerio de Salud Pública y Bienestar Social. Asuncion, Paraguay.

${ }^{4}$ Departamento de Hemato-Oncología Pediátrica, Hospital Central del Instituto de Previsión Social. Asunción. Paraguay.

${ }^{5}$ Servicio de Traumatología Pediátrica, Hospital Central del Instituto de Previsión Social. Asunción. Paraguay.

Correspondencia: Irene Benitez; Correo: irenerocio@gmail.com

Conflicto de Interés: Los autores declaran no poseer conflicto de interés

Recibido: 27/11/2018. Aceptado: 1/04/2019

DOI: https://doi.org/10.31698/ped.46012019007
} 
La histoplasmosis es más frecuente en América del Norte y Central, pero también se encuentra en América del Sur, Asia y África (Figura 1) ${ }^{(2,4)}$. En Paraguay no hay datos epidemiológicos, solo reportes de $\operatorname{casos}^{(6-8)}$.

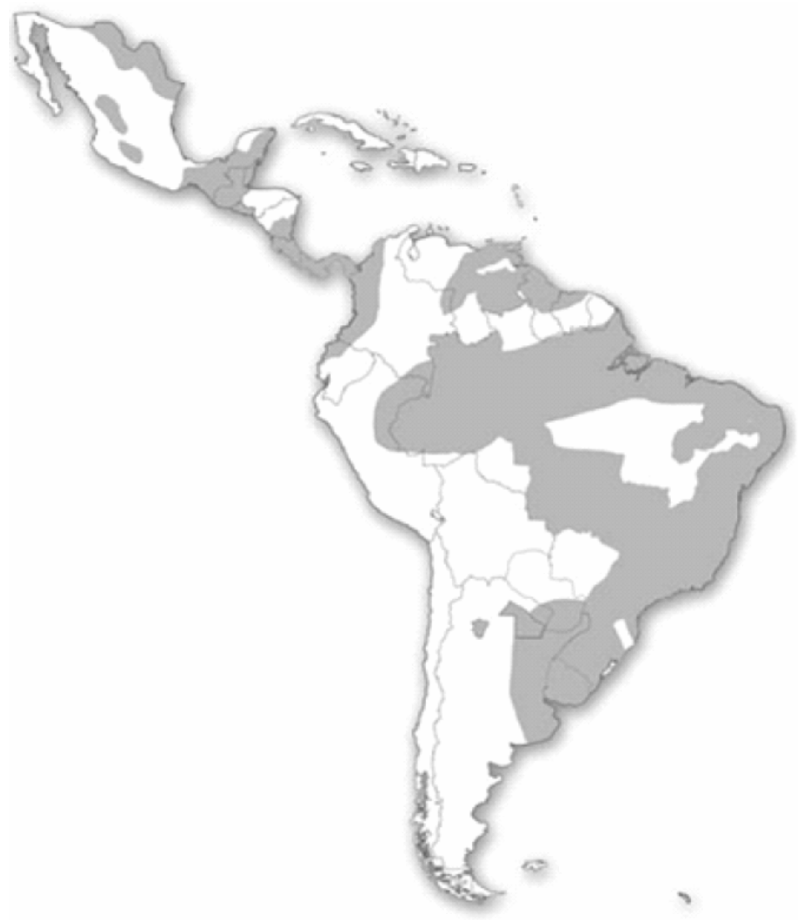

Figura 1. Mapa con distribución de la Histoplasmosis en América Latina ${ }^{(6)}$.

La mayoría de los pacientes expuestos a $H$. capsulatum permanecen asintomáticos o desarrollan solo síntomas leves comunes a otras enfermedades difíciles de diferenciar. La defensa óptima del huésped contra Histoplasma capsulatum requiere interacción entre los macrófagos y las células T. Los pacientes con enfermedades reumáticas que reciben terapias inmunomoduladoras e inmunosupresoras pueden tener un mayor riesgo de histoplasmosis, lo cual ha sido descrita en reportes de casos. La enfermedad diseminada generalmente ocurre en inmunocomprometidos, particularmente en lactantes, infección por VIH, patologías oncológicas, trasplante de órganos sólidos, trasplante de células madre hematopoyéticas, tratamiento con corticosteroides, metotrexato o antagonistas del factor de necrosis tumoral, o deficiencia congénita de células $\mathrm{T}^{(3,4,7)}$.

Los síntomas de la histoplasmosis diseminada incluyen fiebre, malestar general, anorexia y pérdida de peso, pudiendo afectar a varios órganos o ser focalizado, más comúnmente en el sistema reticuloendotelial, la piel, tracto gastrointestinal, intestino, glándula suprarrenal y $\mathrm{SNC}^{(6,7)}$.

Presentamos el caso de un niño con Histoplasmosis, portador de Leucemia Linfoblastica Aguda, quien se encontraba en fase de mantenimiento final en tratamiento con dosis bajas de metrotrexato.

\section{CASO CLÍNICO}

Paciente de 10 años de edad, con diagnóstico de Leucemia Linfoblastica Aguda (LLA) tipo B diagnosticada en agosto 2016. A partir del año 2018 se encontraba en fase de mantenimiento final con Metotrexato $15 \mathrm{mg} / \mathrm{semana}$ por vía oral. Consulta por historia de 3 meses de evolución de lesiones en piel tipo pápulas con posterior ulceración, de $0.5 \mathrm{~cm}$ de diámetro, distribuidas en cara y brazo derecho. Presentó además nódulos subcutáneos periarticulares en región de 2da falange del dedo índice de la mano derecha, metacarpo mano derecha, muñeca derecha y ambos tobillos, dolorosos, con impotencia de funcional y artritis en las articulaciones de las muñecas y los tobillos. Acompaña al cuadro fiebre intermitente, astenia y pérdida de peso. Un mes antes del ingreso aparece pápula con centro ulcerativo, de aproximadamente $1.5 \mathrm{~cm}$ de diámetro en el paladar duro. Fue evaluado por Dermatología que realizo biopsia de piel sin hallazgos significativos, y por Reumatología iniciando tratamiento con prednisona $20 \mathrm{Mg} /$ día ante la sospecha de Atritis Reumatoidea Juvenil por 3 semanas. Ante la falta de respuesta mejoría indico de la lesión del paladar duro; se realizó raspado de la lesión con bisturí y luego coloración con Giemsa del frotis donde se observaron esporos micóticos en semi luna compatibles con Histoplasma capsulatum La biopsia, mostro proceso inflamatorio crónico granulomatoso compatible con Histoplasmosis. (Figura 2).

Con los resultados de las tinciones y de anatomía patológica se inició tratamiento sistémico con Anfotericina B desoxicolato a $1 \mathrm{mg} / \mathrm{kg} /$ día.

El cultivo se realizó en agar Sabouraud dextrosa con 
antibióticos y se incubó a $28^{\circ} \mathrm{C}$ durante 20 días Se obtuvo el crecimiento de colonias blancas de aspecto velloso (Figura 3). En el examen en fresco del cultivo, se observaron macroconidios hialinos, esféricos y tuberculados, característicos de Histoplasma capsulatum (Figura 4 ).

La punción de la medula ósea no demostró signos de recaída de la LLA, ni presencia de gérmenes. Al examen físico pulmones con semiología normal y en abdomen leve hepatomegalia. El fondo de ojo fue normal.

Se realizo TAC de ambas manos, que mostro (Figura 5): lesiones osteolíticas focales y alteración difusa con patrón trabecular en las falanges proximales que interrumpe la cortical ósea del primer y segundo dedos de la mano izquierda Los huesos carpianos mostraron también las mismas lesiones.

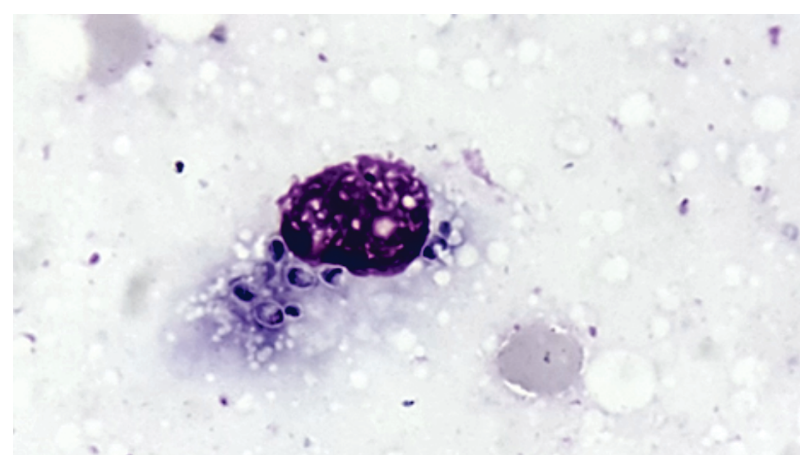

Figura 2. Coloración de Giemsa (1000X): levaduras de Histoplasma capsulatum intracelulares en el interior de un macrófago.

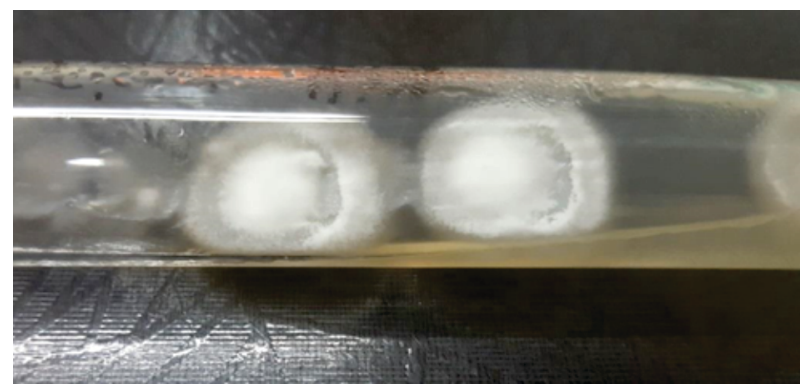

Figura 3. Colonias de Histoplasma capsulatum a $28^{\circ} \mathrm{C}$ en agar Sabouraud dextrosa.

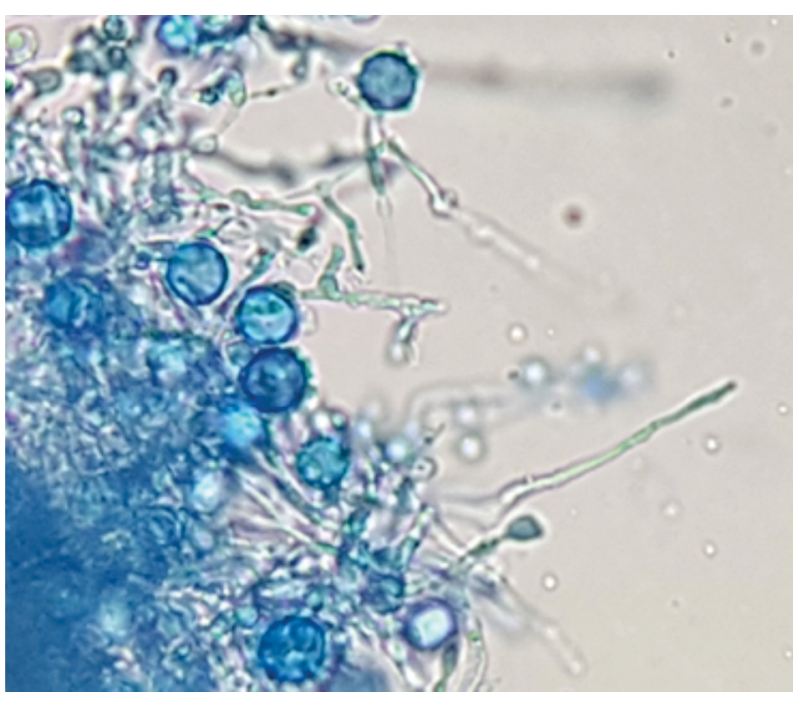

Figura 4. Macroconidios esféricos tuberculados correspondiente a la fase filamentosa de Histoplasma capsulatum.

Recibió tratamiento endovenoso con anfotericina B desoxicolato durante 14 días y luego mantenimiento con itraconazol vía oral durante 12 meses con buena respuesta. (Figura 6).

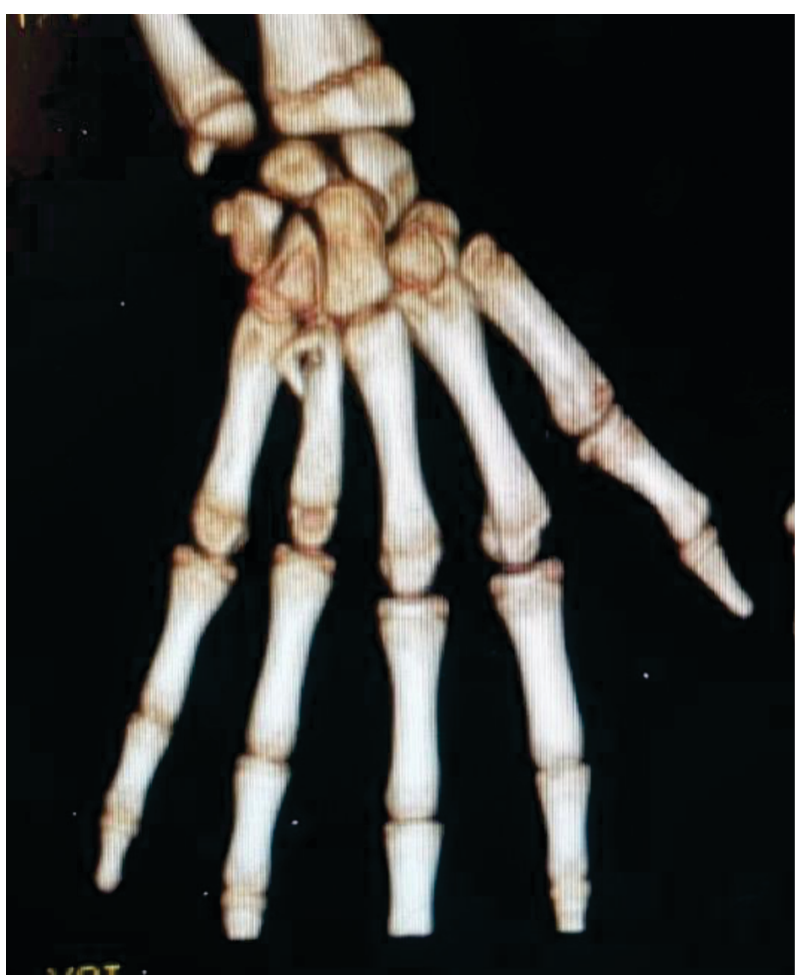

Figura 5. Lesión lítica a nivel de la base del 4to metatarsiano con imagen de secuestro óseo central. 


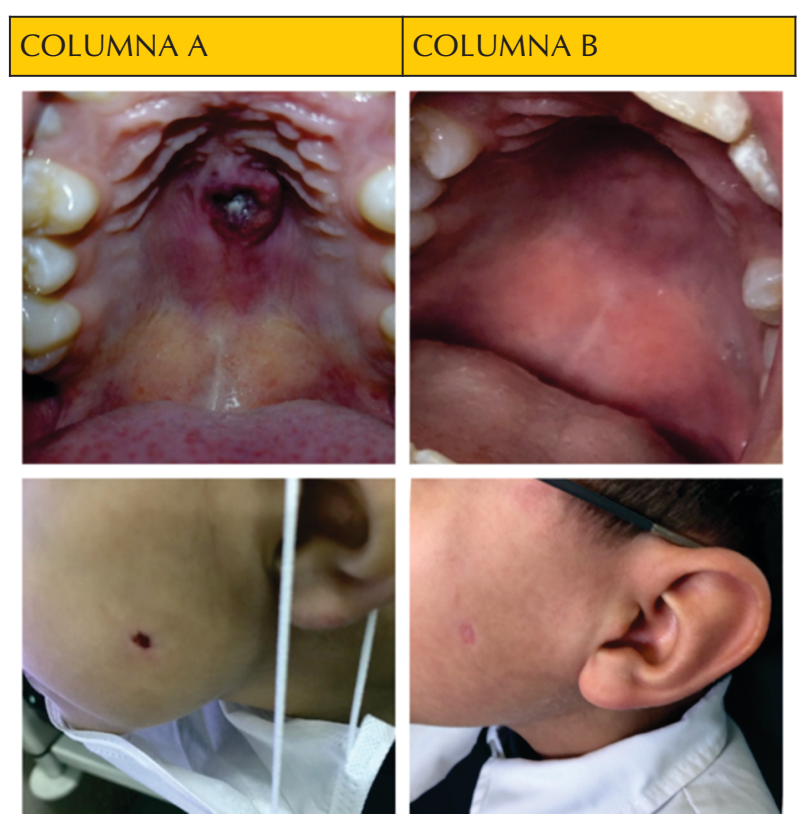

Figura 6. Columna A: Antes del tratamiento. Columna B: Después del tratamiento

\section{DISCUSIÓN}

El presente caso describe a un escolar con diagnóstico de LLA tipo B en fase de mantenimiento, quien desarrollo histoplasmosis diseminada con lesiones cutáneas y afectación ósea, presentación infrecuente de esta enfermedad.

La forma más común de presentación de la histoplasmosis es la diseminada y se describe en pacientes con alteración del sistema inmune pudiendo ocurrir por la exposición exógena o una reactivación de un foco endógeno latente. El diagnóstico implica un desafío por el hecho de no ser frecuente y de compartir sintomatología con otras micosis profundas ${ }^{(3,4)}$.

Las lesiones cutáneas son el resultado de la diseminación hematógena del hongo. Se han reportado en $10-25 \%$ de los pacientes con SIDA con histoplasmosis diseminada. Las lesiones cutáneas son pápulas polimórficas, placas con o sin costras, pústulas, nódulos, úlceras mucosas, erosiones, úlceras perforadas, lesiones que se asemejan a molusco contagioso, erupciones acneiformes, pápulas eritematosas y placas queratósicas, lesiones purpúricas y formas vegetadas localizadas y generalizadas de dermatitis, eritema polimorfo, pioderma gangrenoso, paniculitis, hiperpigmentación difusa, abscesos y celulitis, entre otras ${ }^{(3,7,7,8)}$.

Existen pocos reportes de casos, en su mayoría de adultos, con afectación esquelética por $H$. capsulatum, que incluyen síndrome del túnel carpiano, artritis séptica y osteomielitis. Respecto a la osteomielitis, se describen múltiples lesiones líticas óseas en pacientes con histoplasmosis diseminada, lesiones en el radio distal o huesos del carpo, tibia, peroné y otros En este caso encontramos osteomielitis de las falanges de ambas manos ${ }^{(6,9)}$.

El diagnóstico puede realizarse por estudios micológicos o histopatológicos de las biopsias de las lesiones de la piel o las mucosas como se realizó en el caso descrito. La inmunodifusión en gel de agar y la fijación de complemento, son positivos en más del $90 \%$ de los casos.

En este caso el diagnostico fue realizado mediante el gold estándar que constituye la identificación directa del hongo ${ }^{(10,2,5)}$.

El cribado con la prueba del antígeno de histoplasmaurina en el paciente asintomático parece no ser beneficioso para predecir la infección, por lo que no se recomienda su uso de manera rutinaria.

En las guías de tratamiento de histoplasmosis no se describe el tratamiento de la osteomielitis en particular pero siguiendo las recomendaciones para histoplasmosis diseminada la terapia se inició con anfotericina B (0.7-1 mg / kg / día)) por 2 semanas seguida de itraconazol 12meses. Puede utilizarse también anfotericina B liposomal, Voriconazol y posaconazol

En los pacientes con sida, debe administrarse itraconazol en forma indefinida para prevenir las recidivas, o hasta que el recuento de linfocitos CD4+ sea $>150$ cél $/ \mu l$. Las concentraciones sanguíneas de itraconazol y antígeno de Histoplasma deben ser controladas durante la terapia ${ }^{(11,12)}$. 


\section{REFERENCIAS}

1. Cury GC, Diniz FA, Cruz AG, Hobaika AB. Outbreak of histoplasmosis in Pedro Leopoldo, Minas Gerais, Brazil. Rev Soc Bras Med Trop. 2001;34(5):483-486.

2. Guimaraes AJ, Nosanchuk JD, Zancope-Oliveira RM. Diagnosis of histoplasmosis. Braz J Microbiol. 2006; 37(1):1-13.

3. Leiva M, Cubilla E, Guzmán A, Mendoza G, Aguilar G. Histoplasmosis diseminada aguda en un paciente con infección VIH. Dermatología CMQ. 2012;10(1):28-32.

4. Chena L, Nara E, Canese A, Aguilar G, Araujo P, Taboada A, et al. Coinfección Leishmania infantum, VIH e Histoplasma capsulatum: manifestaciones dermatológicas. Mem. Inst. Investig. Cienc. Salud. [Internet]. 2016 [citado 17 deSep de 2018]; 14(3):121-125.

5. Sepúlveda VE, Márquez R, Turissini DA, Goldman WE, Matute D. Genome sequences reveal cryptic speciation in the human pathogen Histoplasma capsulatum. mBio. 2017; 8(6): e01339-17.

6. Azar MM, Mailinis MF. Case Report: disseminated histoplasmosis with skin lesions and osteomyelitis in a patient from the Philippines. Am J Trop Med Hyg. 2016; 95(1):70-74.
7. Pedroso MCM, de Araújo AB, Crespo AN, Sampaio MH, Sakano E. Nasal septum ulcer as an isolated manifestation of histoplasmosis. ID Cases. 2018; 15: e00490.

8. Pincelli T, Enzler M, Davis M, Tande AJ, Comfere N, Bruce A. Oropharyngeal histoplasmosis: a report of 10 cases. Clin Exp Dermatol. 2019. doi: 10.1111/ced.13927.

9. Palmgren BA, Buhr BR. Histoplasmosis of the tibia. Orthopedics. 2005; 28(1):67-8.

10. Kauffman CA. Histoplasmosis: a clinical and laboratory update. Clin Microbiol Rev. 2007; 20:115-132.

11. Esteban I, Minces P, De Cristofano AM, Negroni R. Histoplasmosis en el sistema nervioso central en una paciente pediátrica inmunocompetente. Arch Argent Pediatr. 2016;114(3):e171-e174.

12. Negroni R, Messina F, Arechavala A, Santiso G, Bianchi M. Eficacia del tratamiento y de la profilaxis antifúngica secundaria en la histoplasmosis asociada al sida: experiencia del Hospital de Infecciosas Francisco J. Muñiz, de la ciudad de Buenos Aires. Rev Ibero am Micol. 2017; 34(2):94-98. 